\title{
Needle Stick or Sharp Injuries \& Associated Factors Among Medical Students at Debre Tabor University
}

\author{
Eleni Girma Woldearegay ${ }^{1, ~ *, ~ B i n i a m ~ E w n t e ~ Z e l e l e w ~}{ }^{2}$ \\ ${ }^{1}$ Department of Medicine, Debre Tabor University, Debra Tabor, Ethiopia \\ ${ }^{2}$ Department of Surgery, Debre Tabor University, Debra Tabor, Ethiopia
}

Email address:

elenigirma99@gmail.com (E. G. Woldearegay)

${ }^{*}$ Corresponding author

\section{To cite this article:}

Eleni Girma Woldearegay, Biniam Ewnte Zelelew. Needle Stick or Sharp Injuries \& Associated Factors Among Medical Students at Debre Tabor University. American Journal of Clinical and Experimental Medicine. Vol. 9, No. 3, 2021, pp. 65-72.

doi: 10.11648/j.ajcem.20210903.13

Received: March 23, 2021; Accepted: May 21, 2021; Published: May 27, 2021

\begin{abstract}
Background: Medical students throughout the world show a high rate of sharp injuries with a consequent risk of acquiring blood-borne infections while performing their clinical activities. Objective: This study was conducted to assess needle stick or sharp injuries among medical students of Debre Tabor University, and to identify the associated factors leading to Needlestick or sharp injury. Method: An institution-based cross-sectional study was conducted among 4th to 6th-year medical students at Debre Tabor University, by using census sampling through a self-administered semi-structured questionnaire, and the completeness of the questionnaires was assessed daily. Data were processed and analyzed using SPSS software version 21.0 and bivariate analysis was obtained using a logistic regression model. In addition, tables and figures were used as necessary to represent the data. Result and conclusion: According to our study, the prevalence of Needle stick or sharp injuries among medical students in clinical years other than clerkship I students in Debre Tabor University was found to be $28.5 \%$. Medical interns were particularly affected with a prevalence rate of $66.6 \%$. The associated factors with Needlestick or sharp injuries included the academic year of study and the department where the injury occurred.
\end{abstract}

Keywords: Occupational Hazard, Medical Interns, Ethiopia, HBV

\section{Introduction}

The National Institute for Occupational Safety and Health, USA defines "Needlestick injuries as injuries caused by needles such as Hypodermic needles, Blood collection Needles, Intravenous (IV) stylets, and Needles used to connect parts of an IV delivery system" [1]. About 35.7 million healthcare workers have a risk of sustaining a needle stick injury worldwide [2].

There are over 20 types of blood-borne pathogens [3, 7]. Among these, it's the transmission of HIV, hepatitis B, and Hepatitis C virus that is of most concern [4].

The World Health Organization has estimated that percutaneous occupational exposure in developing regions account for $40 \%-65 \%$ of hepatitis B (HBV) and hepatitis C virus $(\mathrm{HCV})$ infections [3].

Medical students throughout the world show a high rate of sharp injuries with a consequent risk of acquiring bloodborne infections while performing their clinical activities [1, 3].

Sub-Saharan Africa is a hub of seventy percent of the world's HIV population $[5,13]$. Highly transmittable bloodborne pathogens are vastly prevalent in the health care setting. It is estimated that up to $20 \%$ to $38 \%$ of urban hospitalized patients test positive for a blood-borne pathogen.

Annual reports from U.S. health care workers confer an estimated 600,000 to 800,000 Needlestick and other percutaneous injuries, and there is evidence of vast underreporting of NSSIs among employees in health services $[4,6]$. There is on average 4 NSSI's per worker per year in Africa, Eastern Mediterranean, and Asia according to data from injection safety surveys conducted by the WHO and others [5].

Certain devices can pose an increased risk of injury. They include Disposable syringes $(27 \%)$, suture needles $(25 \%)$, 
and scalpel blades (6\%) among others [7].

Medical students ought to be familiar with exposure risk assessment, management protocols, and the role and risk of Anti-retroviral prophylaxis to prevent the severe consequences of needle stick injuries $[8,14]$.

\section{Statement of the Problem}

The occupational health of 35 million health care workers in the world has long been neglected by institutions as well as governments. This accounts for $12 \%$ of the world's working population [5].

During their clinical practice, health care workers have a risk to acquire viruses with high morbidity and mortality of which the most notable are HBV, HCV, and HIV [9]. The possible exposure risks are regrettably unknown by both patients and health care workers as evidence shows that about 1 in 7 are unaware they have HIV due to lack of testing [7].

Moreover, HBV is known to survive up to a week on objects like discarded needles under optimal conditions [10, 15]. According to CDC 57 documented and 138 possible cases of occupationally acquired HIV infection among healthcare personnel in the united states have been reported since reporting began in 1985 [11].

HCWs incur 2 million NSSIs per year (18) yet this is an underestimation due to lack of surveillance system, and low reporting of NSSIs. Furthermore, almost all of the reported cases of occupational exposure are collected from the developed world whilst over $90 \%$ of the actual exposures take place in the developing nations. Data obtained from WHO shows that on average 4 NSSIs per worker per year occur in the African population [6, 12].

The prevalence of needle stick injuries among Ethiopian Health workers ranges from 13.2 in the Amhara region to $55.1 \%$ in the SNNP region [10].

NSSI prevalence among medical students was found to be $21.4 \%, 22 \%, 39.3 \%, 58 \%$ in Australia, Iran, Mexico, and Germany respectively $[13,14]$. To the best of our knowledge, the prevalence of NSSIs among medical students has not been studied in Ethiopia.

Furthermore, $40-75 \%$ of NSSIs are underreported worldwide [15], and there isn't sufficient data available on Ethiopian medical students on the magnitude and frequency of NSSIs, protective and preventive protocols, and level of reporting.

According to international recommendations, systems for reporting exposures should be arranged by employees to rapidly evaluate the risk of infection [11]. Furthermore, a well-thought-out occupational exposure reporting and intervention system are yet to be studied.

There are compelling reasons to use safer sharps practices, such as the cost of sharps injury. Some of these costs include but are not limited to loss of employee time, cost of laboratory testing, treatment cost for PEP, and cost of reassigning staff [7]. Injuries impose a cost of 175 to 350 USD directly or indirectly on the health care system [16].

\section{Literature Review}

In 1984, the first-ever occupationally acquired HIV infection of a health care worker (HCW) was published launching a new era of concern about the occupational transmission of deadly viruses [17]. All employees in health care settings handling needles or other sharps are at risk for needlestick and sharps injuries (NSSIs).

The center for disease control (CDC) defines a sharp injury as "a penetrating stab wound from a needle, scalpel, or another sharp object that may result in exposure to blood or other body fluids" [7].

An estimated 2 million HCW's experience NSSIs and a risk to acquire infectious diseases each year [18]. The most common blood-borne pathogens from which HCW's are at risk include $\mathrm{HIV}, \mathrm{HBV}$, and $\mathrm{HCV}$ where the estimated risk of infection from a sharp injury is $0.3 \%, 6-30 \%$, and $2-4 \%$ respectively [7]. Health care workers in developing countries have a higher risk of occupational exposure due to the higher prevalence of blood-borne pathogens and lack of safety devices [19]. The current global prevalence of NSI among health workers is estimated to be $44.5 \%$ [16]. However, more than half of percutaneous injuries go unreported so what we have access to be only the tip of the iceberg [7].

Medical interns were found to sustain more needlestick injuries than any other medical students especially during blood-taking practices [2]. A former Intern of Yale University School of medicine had contracted HIV from a percutaneous injury that led to a high-profile lawsuit in the late 1980s is evidence of the devastating consequences of NSSIs [20]. The global prevalence of needle stick injuries among medical interns is estimated to be $42.7 \%$ [18]. The prevalence of NSSI in medical students in the occupied Palestine territory was found to be just over $40 \%$ [9]. Washington University School of medicine reported $30 \%$ of the medical students reported at least one NSI [21], a study conducted in Nigeria shows a $56.9 \%$ of newly graduated Doctors and Dental students have sustained NSI [22].

Some of the factors associated with NSI among final year medical students were socio-demographic backgrounds, duration of exposure, knowledge, and perception of risk of Bloodborne pathogens, and level of practice of universal precautions [1].

The education of health care workers and minimization of invasive procedures are some of the strategies that are available to prevent infections due to sharp injuries. In addition, the use of safer devices also plays a paramount role in preventing injuries from sharp items [23]. Safer techniques can also be applied to prevent most NSSI such as avoiding recapping needles by hand, appropriate disposal of sharp items, and using medical equipment with safety enhancement features [7].

Employers and workers should be familiar with universal (UP), standard (SP), and transmission-based precautions (TBP). The former is an approach in which all human blood and body fluids are treated as potentially infectious while SP is applied to all who are not known or suspected to be 
infectious by practicing hand hygiene and use of PPE, safe injection practices, and safe management of contaminated objects. TBP augments SP with additional measures to interrupt the route of transmission that is based on what is already known or suspected about a patient [24].

The hierarchy of controls concept is a model used by health care organizations in recent years to prioritize interventions to prevent occupational injuries by applying engineering control where the priority is to reduce the use of needles and other sharps where possible, and the next is to isolate the hazard through the use of sharp disposal containers and devices with integrated engineered sharps injury prevention feature. If these strategies are not available, the focus shifts to work-practice controls and PPE [7, 8, 18].

\section{Justification of the Study}

As part of the budding workforce in health care settings, medical students and medical interns are a valuable asset to the health care community; yet, peer reviews indicate a noticeably high number of NSSI among medical students with potential drastic health consequences. To the best of our knowledge, the prevalence of NSSI among medical students has not been studied in Ethiopia. As a result, we find it crucial to assess the magnitude of medical students' exposure to deadly infections and find solutions to mitigate the problem. Moreover, it is our belief this study will serve as a blueprint for similar studies to be conducted in the future.

\section{Objectives}

\subsection{General Objective}

To assess needle stick or sharp injuries and their associated factors among medical students in Debre Tabor University.

\subsection{Specific Objectives}

1) To determine the magnitude of a needle stick or sharp injuries among medical students in DTU

2) To identify the factors leading to needle stick injury among medical students in DTU

\section{Materials and Methods}

\subsection{Study Area and Period}

The study will be conducted in Debre Tabor University, College of health science, located in Debre Tabor town, the capital city of South Gondar Zone. It is situated $98 \mathrm{~km}$ to the east of Bahirdar, the capital of the Amhara region, and about $667 \mathrm{~km}$ from Addis Ababa which is the capital city of Ethiopia. The university was established in $2011 \mathrm{G}$; currently, 246 medical students are enrolled in its Hybrid innovative curriculum. Excluding pre-clerkship I students. 144 students are currently enrolled in the academic years $4^{\text {th }}$ to $6^{\text {th }}$.

This study was conducted from October 22 to January 18 , 2021.

\subsection{Study Design}

Institutional-based cross-sectional study design.

\subsection{Source Population}

Medical students in Debre Tabor University, College of health sciences in the academic year 2012 -2021.

\subsection{Study Population}

All eligible Medical students attending their clerkship studies (Clerkship II, III), and final year medical students or medical Interns (MI's) at Debre Tabor University, College of health sciences in the academic year 2012 -2021. Given the current pandemic clerkship, I students were not available on school grounds so they could not be included in this study.

\subsection{Sampling Technique}

Census sampling technique

\subsection{Eligibility}

\subsubsection{Inclusion Criteria}

1) All students who are enrolled in clerkship studies, and

2) Final year medical students will be included in this study

\subsubsection{Exclusion Criteria}

Non-visibly bloody solutions (tears, urine, feces) will be excluded from this study

\section{Data Collection and Analysis}

\subsection{Data Collection Tools and Procedures}

Before starting the actual data collection the objective or purpose of the study was clearly described for the participants, informed consent was obtained from all selected volunteer students. Subsequently, they were provided via self-administered semi-structured questionnaires in the official learning language of the university.

Data were collected using a modified self-administered semi-structured questionnaire derived from EPINET Needlestick and sharp Injury report form.

\subsection{Data Quality and Control}

After a letter of cooperation is obtained from Debre Tabor University, college of health sciences. The completeness of the questionnaire was assessed daily, and the overall quality of the data was monitored.

\subsection{Data Processing and Analysis}

The data was categorized under separate titles for the benefit of reporting. Data processing and analysis were done using SPSS VERSION 21.0. Binary logistic regression analysis was applied to find an association between the predictor and the outcome variable and a variable with $\mathrm{P}$ value $<0.25$ in bi-variable logistic regression analysis was 
included in multivariate logistic regression analysis. A Pvalue $<0.05$ was considered significant, and an odds ratio with a 95\% confidence interval was used to examine associations between predictors and outcome variables. Furthermore, tables and figures with their respective descriptions as necessary are depicted.

\subsection{Operational Definition}

Hollow-bore needle: Needle (e.g., hypodermic needle, phlebotomy needle) with a lumen through which material (e.g., medication, blood) can flow [8]

Needlestick/sharp Injury - a penetrating stab wound from a needle, scalpel, or other sharp objects that may result in exposure to blood or other body fluids

Potentially preventable injury - if a needle was unnecessarily used, a device's safety feature was not activated or was used improperly, a conventional device was used instead of a market available safety device, a safer work environment might have prevented the injury, or a sharp was disposed of improperly.

Patient-care related injury - when none of the aforementioned conditions apply, injuries less amenable to the promotion of safer routine work practices and technologies

Personal protective equipment -These include items such as gloves, safety glasses and shoes, earplugs or muffs, hard hats, respirators, or coveralls, vests, and full-body suits worn to minimize exposure to serious workplace injuries and illnesses resulting from contact with chemical, radiological, physical, electrical, mechanical or other workplace risks

Standard Precautions: An approach to infection control recommended by the Centers for Disease Control and Prevention since 1996. Standard precautions synthesize the major features of universal precautions and apply to blood and all moist body substances, not just those associated with blood-borne virus transmission. Standard precautions are designed to prevent transmission of infectious agents in the healthcare setting to patients and healthcare personnel [8].

Superficial NSSIs - little or no bleeding

Moderate NSSIs - skin punctured, some bleeding

Severe NSSIs - deep stick/cut, or profuse bleeding

Universal Precautions: An approach to infection control that treats all human blood and other potentially infectious materials as if they were infectious for HIV and HBV or other bloodborne pathogens [8].

\section{Result}

There were a total of 144 medical students in the academic year belonging from the fourth year to the final year. Among them, 47 were from Clerkship II, 55 from Clerkship III, and 42 medical interns participated in this study which gave a response rate of $100 \%$.

Out of the total of 144 medical students, 41 (28.5\%) have sustained needle stick/sharp injury (figure 1). Among them, $28(66.6 \%)$ of medical interns, $10(18.1 \%)$ of clerkship III students, and $4(8.5 \%)$ of clerkship II students have sustained
NSSIs.

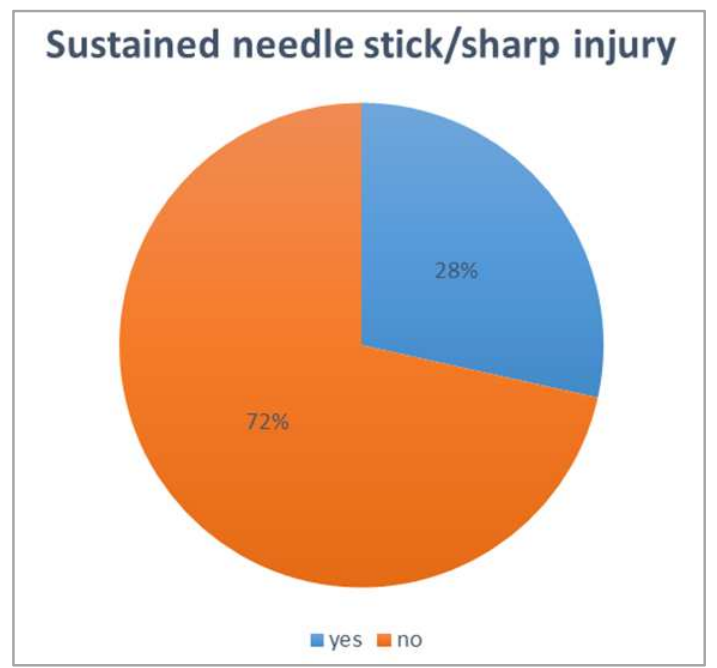

Figure 1. Students who sustained needle stick/sharp injury in Debre Tabor University, college of health sciences, 2020.

Around $73 \%$ of the NSSIs occurred in the department of surgery followed by the department of gynecology and obstetrics (21.9\%) (Table 1).

Table 1. Department where needle stick/sharp injury has occurred among medical students in Debre Tabor University, college of health sciences, December 2020.

\begin{tabular}{lll}
\hline Department where NSSI occurred & Frequency & Percent \\
\hline Surgery & 30 & 73.1 \\
Internal medicine & 1 & 2.4 \\
Paediatrics & 1 & 2.4 \\
Gynaecology and Obstetrics & 9 & 21.9 \\
Total & 41 & 100.0 \\
\hline
\end{tabular}

The current study reveals most of the NSSIs occurred by the patient's bedside in $15(10.4 \%)$ Of the case (Table 2$)$.

Table 2. Place where NSSIs occurred in Debre Tabor University, College of Health Sciences, December 2020.

\begin{tabular}{lll}
\hline Place where NSSIs occurred & Frequency & Percent \\
\hline Patient bedside & 15 & 10.4 \\
Procedure room & 8 & 5.6 \\
Emergency room & 8 & 5.6 \\
Operating room & 5 & 3.5 \\
Labor and delivery room & 5 & 3.5 \\
Total & 41 & 100.0 \\
\hline
\end{tabular}

Among the 41 students who had sustained needle stick/sharp injury, $33(22.9 \%)$ claim the source patient was identifiable, whilst 6 students $(4.2 \%)$ claim otherwise, and for the rest $1.4 \%$, the question was not applicable.

Concurrently, 31 of the injured students were the user of the sharp item while 10 students were not. Of the devices that caused the injury $32(22.2 \%)$ were during their first use and 8 $(5.6 \%)$ were during re-use of the device and for 1 device its usage status is unknown.

Among the 41 NSSIs, 22 were with contaminated items (Figure 2). 


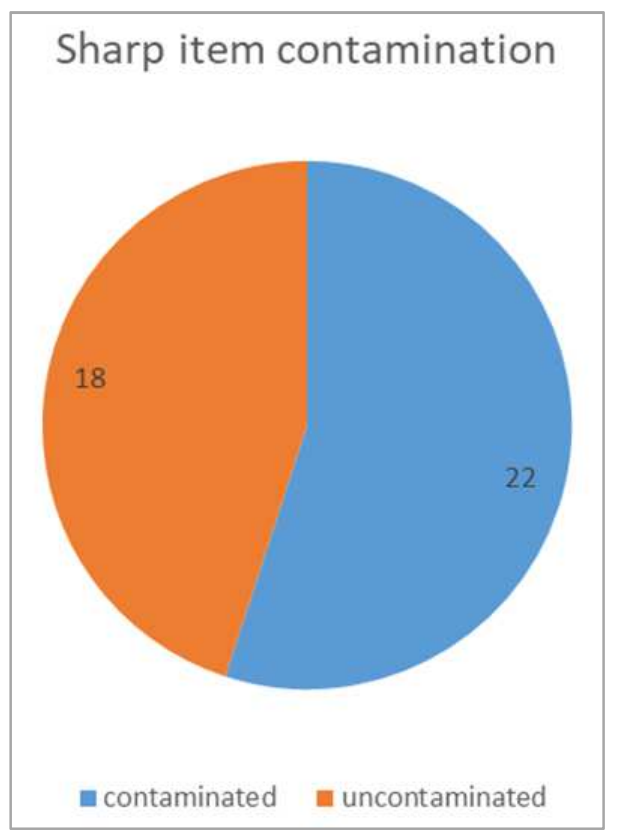

Figure 2. Sharp item contamination status of NSSIs in DTH, College of Health Science, December 2020.

Of the 41 students who sustained needle stick/sharp injury 13 had noted blood on the device. The purpose the sharp item was being used initially was for suturing in 9 $(6.3 \%)$ of the cases, Injection/intramuscular or subcutaneous in $8(5.6 \%)$, finger stick/heel injury in 6
(4.2\%), for cutting in $4(2.8 \%)$, to place an arterial/central line and to flush IV line in $3(2.1 \%)$ of cases each, to draw venous blood and to contain specimen/glass item in 2 $(1.4 \%)$ of cases each, and lastly to start IV infusion in 1 of the cases.

The devices that caused NSSIs include needle on the disposable syringe in $10(6.9 \%)$ of the cases, hollow needle, suture needle and syringe needle in $8(5.6 \%)$, spinal or epidural needle in $2(1.4 \%)$, and Towel clip, glass item, vacuum tube blood collection, and needle not sure what kind each consisting of 1 case $(0.7 \%$ each $)$.

Most of the injuries $22(15.3 \%)$ happened during the use of the item followed by before use of the item in $13(9 \%)$ (Table 3).

Table 3. Time of NSSIs in DTH, December 2020.

\begin{tabular}{lll}
\hline Time of NSSIs & Frequency & Percent \\
\hline $\begin{array}{l}\text { Before the use of the item } \\
\text { the device left on floor, table, bed, or other }\end{array}$ & 13 & 9.0 \\
inappropriate places & 1 & .7 \\
$\begin{array}{l}\text { During use of the item } \\
\text { After use or before disposal }\end{array}$ & 22 & 15.3 \\
While putting item near disposal container & 3 & 2.1 \\
In preparation for re-use & 1 & .7 \\
Total & 1 & .7 \\
\hline
\end{tabular}

The location of the disposal container was within arm's reach in $20 \%$ of cases and a different room in around $11 \%$ (Figure 3).

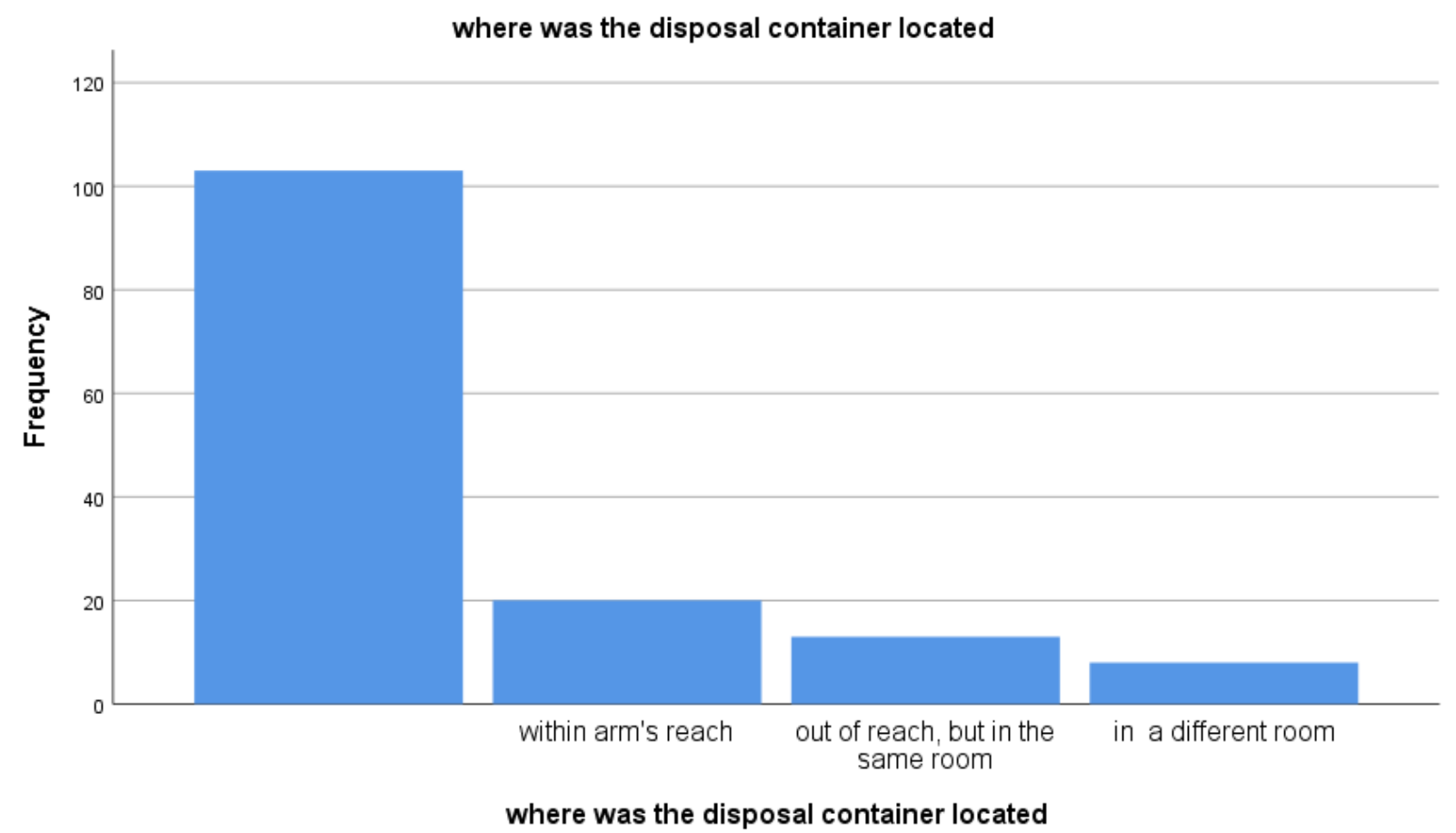

Figure 3. Location of the sharp item disposal container in DTH, December 2020.

Of the 41 needle stick/sharp injuries, 26 (18.1\%) occurred on the right hand, and $15(10.4 \%)$ occurred on the left hand. Of these injuries, $19(13.2 \%)$ were superficial, and 22 $(15.3 \%)$ were moderate injuries. None of the injuries were severe.
At the time of injury, $24(16.7 \%)$ were wearing a single pair of gloves, $13(9 \%)$ were wearing double pair of gloves and $4((2.8 \%)$ were not wearing gloves.

Out of the 144 medical students included in this study, 112 $(77.8 \%)$ have received full dose of Hepatitis $\mathrm{B}$ vaccination, 
while $26(18 \%)$ have received partial doses and $6(4.2 \%)$ have not received the vaccine.

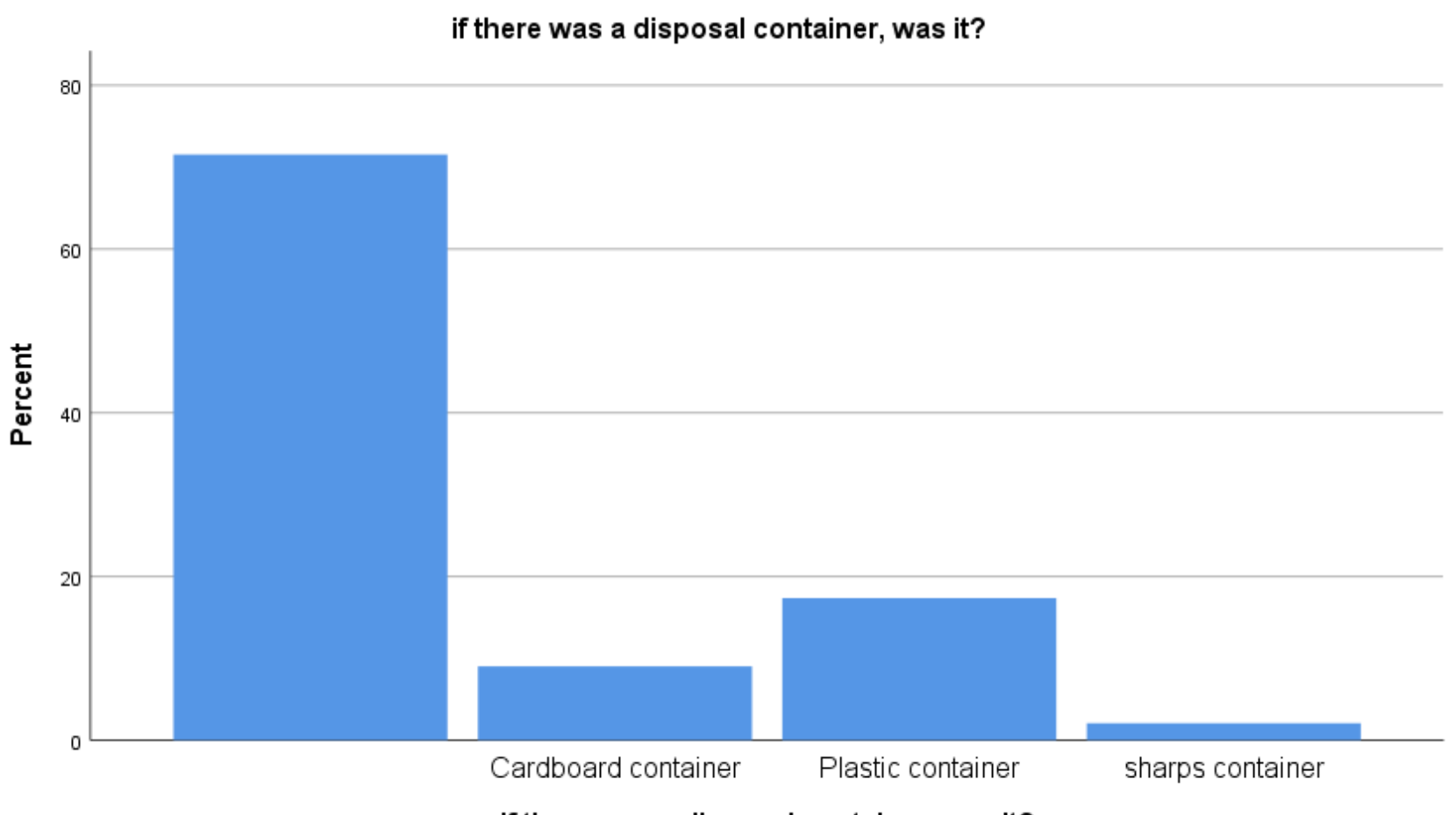

if there was a disposal container, was it?

Figure 4. Material disposal waste container is made of in DTH, December 2020.

Factors associated with Needlestick/sharp injury

Table 4. Factors associated with NSSIs among medical students in DTU, December 2020.

\begin{tabular}{llll}
\hline Variables & Crude odds ratio with 95\% CI & Adjusted odds ratio with 95\% CI & P-value \\
\hline Year of study & $9.00(3.52-23.0)$ & $4.228(9.204-22.749)$ & $<0.001$ \\
Department where injury occurred & $10.21(2.25-46.18)$ & $2.012(7.02-9.056)$ & 0.02 \\
Time of injury & 0.00 & & 0.99 \\
Number of gloves used & $1(000)$ & & 0.99 \\
\hline
\end{tabular}

In the multivariate analysis, the academic year of study and department where the injury occurred was found to be highly associated with the occurrence of NSSIs among medical students.

\section{Discussion}

The prevalence of NSSIs among medical students was found to be $28.5 \%$ in our study which is higher than $7 \%$, $21.4 \%, 22 \%, 23 \%$ prevalence rates in Malaysia, Australia, New York, and Germany respectively $(3,13,4,20)$. These higher prevalence rates can be explained by the difference in utilizing resources for universal precautions between developing and developed countries. The value of the current study was lower in comparison to $30 \%, 39.3 \%, 41.2 \%$ studies conducted in Missouri, Iran, and Palestine respectively $(5,14,10)$. The reasons attributed to lower prevalence rates could lie in the difference in the total number of medical students and the difference in the working environment.

This study shows a higher prevalence rate of NSSIs among medical interns $66.6 \%$ as compared to students in lower grades.
Similarly, a study in Germany notes the higher prevalence of NSSIs among final year medical students or medical interns since they are frontline in the task of blood-taking practice in comparison to their juniors (20).

Half of NSSIs occurred during the use of the item which is per a study in Germany (20) which signifies that the main risk factor for NSSI is learning manual procedures.

The present study revealed that most of the NSSIs (73\%) occurred in the department of Surgery followed by the department of gynecology and obstetrics which is comparable with a study done in Palestine (10). The aforementioned departments are prone to NSSIs because they are predominated by procedural skill tasks involving many sharp items including needles.

The most common device responsible for NSSIs in this study were found to be needles (needle on disposable syringe, hollow needle, suture needle) which congregates with studies done in Malaysia (1).

Multivariate analysis results in this study reveal the risk of developing NSSIs for medical interns were 4 times higher than C II students (AOR 4.2) which is comparable to a study in Palestine which states the relationship between education 
level (medical Interns Versus students) and NSSIs were statistically significant ( $\mathrm{p}$-value $<0.001$ ). Moreover, NSSIs were also found to occur 2 times more in the surgical department as compared to other departments in this study.

The majority of the injuries in this study were self-inflicted similar to studies in Malaysia (3) and Germany (16)

Most of the respondents in this study are fully vaccinated against Hepatitis B (77.8\%) which is lower compared to $96.8 \%$ in Iran (14), and 93\% in Malaysia (3) the reasons for lower values in this study pertain to lack of availability of the vaccine in school grounds, and high cost of the vaccine.

\section{Conclusion}

NSSIs are found to be highly prevalent among medical students in the clinical years of their studies at Debre Tabor University. Final year medical students or medical interns are particularly affected owing to long working hours.

The factors found to be highly associated with the occurrence of NSSIs in this study include academic year and department where the injury occurred.

\section{Acronyms and Abbreviations}

\author{
$\mathrm{BBF}$ - blood and body fluids \\ CDC - Center for Disease Control \\ DTH - Debre Tabor Hospital \\ DTU- Debre Tabor University \\ EPINET - Exposure Prevention Information Network \\ HBV - Hepatitis B Virus \\ HCV - Hepatitis C Virus \\ HCW - Health Care Worker \\ HIV - Human Immune deficiency Virus \\ IV - Intravenous \\ NIOSH - National Institute for Occupational Safety and \\ Health \\ NSSIs - Needle stick sharp injuries \\ USD - United States Dollar
}

\section{Ethical Consideration}

Before engaging in the data collection process, the Community based education coordinating office had asked permission from Debre Tabor University through a letter of permission. Furthermore, before commencing data collection, we were obliged to acquire the consent of the respondents after explaining the importance of their participation and receive verbal consent from the respondents.

\section{Disclosure}

The authors report no conflicts of interest in this work.

\section{Consent for Publication}

All the students who filled the questionnaire have consented to the publication of this work via a consent form attached with the questionnaires. The Authors could provide these upon request.

\section{Data Availability}

All the data supporting the results reported in the manuscript can be found from the authors.

\section{Funding}

The authors have not received any funding to conduct this work.

\section{Competing Interests}

The authors declare there are no competing interests to conduct this work.

\section{Acknowledgements}

The medical students of Debre Tabor University are the core upon which this paper is built, and we would like to send a big thanks to their way.

\section{References}

[1] Norsayani, M. Y. and I. N. J. J. o. o. h. Hassim, Study on incidence of needle stick injury and factors associated with this problem among medical students. 2003. 45 (3): p. 172-178.

[2] Deisenhammer, S., et al., Needlestick injuries during medical training. 2006. 63 (3): p. 263-267.

[3] Swe, K. M. M., et al., The prevalence of needle sticks injury among medical students in Melaka, Malaysia, A cross sectional study. 2012. 71 (2): p. 214-220.

[4] Choi, L. Y., et al., Sharps and needlestick injuries among medical students, surgical residents, faculty, and operating room staff at a single academic institution. 2017. 74 (1): p. 131-136.

[5] Wilburn, S. Q., G. J. I. j. o. o. Eijkemans, and e. health, Preventing needlestick injuries among healthcare workers: a WHO-ICN collaboration. 2004. 10 (4): p. 451-456.

[6] Sharma, G. K., et al., Needlestick injuries among medical students: incidence and implications. 2009. 84 (12): p. 18151821.

[7] 20195 May 2021]; Available from: https://www.cdc.gov/nora/councils/hcsa/stopsticks/default.htm l\#: :text=The $\% 20$ Stop $\% 20$ Sticks $\% 20$ campaign $\% 20$ is,and $\% 2$ 0other\%20sharps $\% 20$ related $\% 20$ injuries.

[8] 2021 May 20215 may 2021]; Available from: http://www.mtpinnacle.com/.

[9] Abu-Rmeileh, N. and M. Al-Dabbas, Needlestick injury among interns and medical students in the Occupied Palestinian Territory. 2012.

[10] Kebede, G., M. Molla, and H. R. J. S. S. Sharma, Needle stick and sharps injuries among health care workers in Gondar city, Ethiopia. 2012. 50 (4): p. 1093-1097. 
[11] Notes from the Field: Occupationally Acquired HIV Infection Among Health Care Workers - United States, 1985-2013. 20155 May 2015]; Available from: https://www.cdc.gov/mmwr/preview/mmwrhtml/mm6353a4.h tm?s_c.

[12] Ghasemzadeh, I., et al., Sharp injuries among medical students. 2015.7 (5): p. 320.

[13] Cossart, Y. E. and B. J. M. j. o. A. deVries, Needlestick injury in medical students. 1994. 160 (7): p. 398-400.

[14] Lauer, A.-C., et al., Needlestick and sharps injuries among medical undergraduate students. 2014. 42 (3): p. 235-239.

[15] Daley, K. 2004; Available from: https://www.who.int/occupational_health/activities/5prevent.p df.

[16] Bouya, S., et al., Global prevalence and device related causes of needle stick injuries among health care workers: a systematic review and meta-analysis. 2020. 86 (1).

[17] Wanchu, A., et al., Possible occupationally acquired HIV infection in two Indian healthcare workers. 2006.8 (2): p. 56.

[18] Available

from: https://www.who.int/occupational health/topics/needinjuries/e $\mathrm{n} /$.

[19] Auta, A., et al., Health-care workers' occupational exposures to body fluids in 21 countries in Africa: systematic review and meta-analysis. 2017.95 (12): p. 831.

[20] Cervini, P. and C. J. J. o. g. i. m. Bell, Brief report: needlestick injury and inadequate post-exposure practice in medical students. 2005. 20 (5): p. 419-421.

[21] Patterson, J. M. M., et al., Needlestick injuries among medical students. 2003. 31 (4): p. 226-230.

[22] Osazuwa-Peters, N., et al., Occupational exposure to sharp injuries among medical and dental house officers in Nigeria. 2013. 26 (2): p. 283-290.

[23] Wicker, S., et al., Prevalence and prevention of needlestick injuries among health care workers in a German university hospital. 2008. 81 (3): p. 347-354.

[24] Worker protections against occupational exposure to infectious diseases. Available from: https://www.osha.gov/bloodborne-pathogens/workerprotections. 